\title{
Seleção de Descritores na Caracterização de Germoplasma de Paspalum sp. por meio de Componentes Principais ${ }^{1}$
}

\author{
Elizabeth Strapasson ${ }^{2}$, Roland Vencovsky ${ }^{3}$, Luiz Alberto Rocha Batista ${ }^{4}$
}

\begin{abstract}
RESUMO - Foram avaliados 15 descritores reprodutivos, 22 vegetativos e 21 agronômicos, em que os agronômicos compreenderam sete descritores para a avaliação anual, sete descritores para a avaliação de inverno e sete descritores para a avaliação de verão, com o objetivo de selecionar os melhores descritores botânico-agronômicos para caracterizar acessos das espécies Paspalum guenoarum e Paspalum plicatulum. Utilizou-se a análise de componentes principais para descartar os descritores considerados redundantes ou nãodiscriminantes. Este método permitiu redução de 53, 68 e 43\% dos descritores reprodutivos, vegetativos e agronômicos, respectivamente, do conjunto inicialmente considerado. Foi possível selecionar, por intermédio desta técnica, oito descritores, considerados mais importantes na descrição da variabilidade presente na coleção de acessos do germoplasma estudado.
\end{abstract}

Palavras-chave: acessos, análise de componentes principais, germoplasma, seleção de descritores

\section{Selection of Descriptors for Paspalum sp. Germplasm Characterization by Means of Principal Components}

\begin{abstract}
Fifteen reproductive, 22 vegetative and 21 agronomic descriptors were evaluated, the agronomic ones comprised seven descriptors for annual, winter and summer evaluations, respectively, with the objective to select the most appropriate descriptors for characterization of Paspalum guenoarum and Paspalum plicatulum. Principal components analysis was used to discard redundante or non-discriminative descriptors. The principal component analysis allowed a reduction of 53, 68 and 43\%, respectively, in the number of initial descriptors considered. By means of this technique, it was possible to select eight descriptors, considered the most important descriptors to identify the genetic variability in this germplasm collection.
\end{abstract}

Key Words: accessions, germplasm, principal components analysis, selection descriptors

\section{Introdução}

Atualmente, a pecuária brasileira é sustentada por um número muito restrito de espécies forrageiras. Esta estreita base genética limita a capacidade de se elevarem os patamares de produtividade da pecuária brasileira, o que a torna vulnerável aos ataques de pragas e/ou doenças às quais estas espécies possam ser susceptíveis.

O gênero Paspalum compreende mais de 400 espécies tropicais e subtropicais, cuja importância é evidenciada por sua adaptabilidade a diferentes ecossistemas, o que representa menor risco de causar desequilíbrio biológico devido à grande diversidade genética existente.

Esta diversidade apresentada pelas espécies do gênero Paspalum levou CHASE (1929) a propor sua divisão em grupos taxonômicos. Dentre estes grupos, o de maior importância no Brasil Central é o Plicatula, podendo-se destacar, pelo seu potencial forrageiro, as espécies Paspalum guenoarum e Paspalum plicatulum.

O Centro de Pesquisa de Pecuária do Sudeste, CPPSE/EMBRAPA - São Carlos - São Paulo, vem realizando estudos de caracterização e avaliação agronômica no germoplasma dessas espécies, com o objetivo de obter novas variedades de forragem.

Dessa forma, buscou-se neste trabalho selecionar os descritores que melhor se prestam para caracterizar acessos das espécies Paspalum guenoarum e Paspalum plicatulum, eliminando aqueles considerados redundantes.

\footnotetext{
${ }^{1}$ Parte da Dissertação apresentada pelo primeiro autor ao Departamento de Matemática e Estatística, ESALQ-USP, para obtenção do grau de Mestre.

2 Professor do Departamento de Matemática Aplicada da Universidade Estadual de Londrina, Caixa Postal 6001 Campus Universitário, CEP 86051 990 - Londrina-PR - FAX (043) 371-4216. E-mail: estrapas@uel.br

${ }^{3}$ ESALQ-USP, Departamento de Genética, Caixa Postal 83, CEP: 13400-970, Piracicaba-SP. E-mail: vencovsky@zaz.com.br

${ }^{4}$ Pesquisador do Centro de Pesquisa de Pecuária do Sudeste-CPPSE/EMBRAPA, Rodovia Washington Luiz km 234, Caixa Postal 339, CEP 13560970, São Carlos-SP. E-mail: Ibatista@cppse.embrapa.br
} 


\section{Material e Métodos}

O estudo envolveu 27 acessos do gênero Paspalum, pertencentes ao grupo Plicatula, sendo cinco da espécie Paspalum guenoarum e 22 da espécie Paspalum plicatulum.

As caracterizações botânico-agronômicas foram realizadas em três ensaios no delineamento experimental em blocos ao acaso, com três repetições. A primeira repetição de cada ensaio foi utilizada para avaliação dos caracteres reprodutivos e vegetativos, e as outras duas foram utilizadas para avaliação agronômica. O número total de parcelas foi 81 , com dimensões de $5 \mathrm{~m} \times 2 \mathrm{~m}$ e as mudas espaçadas entre si de $0,5 \mathrm{~m} \times 0,5 \mathrm{~m}$, com área útil de $4 \mathrm{~m} \times 1 \mathrm{~m}$, constituída pelas duas fileiras centrais.

Os ensaios 1 e 2 contaram com 20 acessos. A avaliação agronômica foi realizada por intermédio de quatro cortes sucessivos, sendo que da soma dos quatro cortes foi obtida a avaliação anual dos dois primeiros cortes a avaliação de inverno e dos dois últimos, a avaliação de verão.

O ensaio 3 contou com sete acessos, e sua avaliação agronômica anual procedeu-se como citado acima. A avaliação de inverno foi a soma dos cortes um e quatro e a avaliação de verão, a soma dos cortes dois e três.

Os seguintes descritores botânico-agronômicos foram avaliados:

A) parte reprodutiva: pilosidade do escapo (PE), pilosidade do eixo floral (PEF), pilosidade do rácemo (PR), pilosidade da espigueta (PES), disposição do rácemo no eixo floral (DREF), época de florescimento (EFL), época de maturação (EM), comprimento do escapo (CE), comprimento do eixo floral (CEF), número médio de rácemo por inflorescência (NMRI), número médio de pedicelos por rácemo (NMPR), comprimento médio do rácemo mais longo (CMRML), comprimento médio da semente (CMS), largura média da semente (LMS) e número médio de sementes em 1 grama (NMS).

B) parte vegetativa: número médio de entrenós (NMEN), comprimento médio dos entrenós (CMEN), altura média das plantas (AMP), distribuição dos pêlos no limbo foliar (DIPLF), densidade dos pêlos no limbo foliar (DEPLF), comprimento dos pêlos no limbo foliar (COPLF), distribuição dos pêlos nas bainhas (DIPB), densidade dos pêlos nas bainhas (DEPB), comprimento dos pêlos nas bainhas (COPB), densidade dos pêlos nos nós (DEPN), comprimento dos pêlos nos nós (COPN), distribuição dos pêlos nos entrenós (DIPEN), densidade dos pêlos nos entrenós (DEPEN), comprimento dos pêlos nos entrenós (COPEN), comprimento médio da lâmina foliar da base (CMLF), largura média da lâmina foliar da base (LMLFBASE), largura média da lâmina foliar do meio (LMLFMEIO), comprimento médio da bainha (CMB), largura média da base da bainha (LMBBASE), largura média do meio da bainha (LMBMEIO), largura média do ápice da bainha (LMBAPICE) e comprimento da haste reprodutiva (CHR).

C) parte agronômica:

avaliação anual: porcentagem média de matéria seca (PORMSANO), produção total de matéria seca $(\mathrm{kg} / \mathrm{ha})$ (PRMSANO), porcentagem média de proteína bruta na matéria seca (PPMSANO), porcentagem média de fibra em detergente neutro (FDNANO), porcentagem média de digestibilidade in vitro (DIVANO), nota média de desenvolvimento vegetativo (NOTANO) e rebrota média (REBROAN).

avaliação de inverno: porcentagem média de matéria seca (PORMSINV), produção total de matéria seca (kg/ha) (PRMSINV), porcentagem média de proteína bruta na matéria seca (PPMSINVE), porcentagem média de fibra em detergente neutro (FDNINVER), porcentagem média de digestibilidade in vitro (DIVINVER), nota média de desenvolvimento vegetativo (NOTAINV) e rebrota média (REBROIN).

avaliação de verão: porcentagem média de matéria seca (PORMSVER), produção total de matéria seca (kg/ha) (PRMSVER), porcentagem média de proteína bruta na matéria seca (PPMSVER), porcentagem média de fibra em detergente neutro (FDNVERAO), porcentagem média de digestibilidade in vitro (DIVERAO), nota média de desenvolvimento vegetativo (NOTAVER) e rebrota média (REBROVER).

Todas as características reprodutivas, vegetativas e agronômicas foram avaliadas de acordo com padrões estabelecidos pelo CPPSE/EMBRAPA.

Utilizou-se a técnica de componentes principais para verificar a possibilidade de descartar os descritores não-discriminantes.

A metodologia da análise de componentes principais trata-se de uma técnica puramente matemática e encontra-se melhor descrita em MORRISON (1976), MARDIA et al. (1979) e JOHNSON e WICHERN (1982). A análise de componentes principais é um método multivariado que consiste em transformar um conjunto de $\mathrm{p}$ variáveis originais $\mathrm{X}_{1}, \mathrm{X}_{2}, \ldots$, 
$\mathrm{X}_{\mathrm{p}}$, pertencentes a $\mathrm{n}$ indivíduos ou populações, em um novo conjunto de variáveis, $\mathrm{Y}_{1}, \mathrm{Y}_{2}, \ldots, \mathrm{Y}_{\mathrm{p}}$ de dimensão equivalente, chamados componentes principais.

Cada componente principal é uma combinação linear das variáveis originais, construído de maneira a explicar o máximo da variabilidade total dessas variáveis originais e não-correlacionado entre si.

MORRISON (1976) define o primeiro componente principal $\left(\mathrm{Y}_{1}\right)$ de um conjunto de $\mathrm{p}$ variáveis, $\mathrm{X}_{1}, \mathrm{X}_{2}, \ldots, \mathrm{X}_{\mathrm{p}}$, contidas no vetor $\mathrm{X}^{\prime}=\left(\mathrm{X}_{1}, \mathrm{X}_{2}, \ldots, \mathrm{X}_{\mathrm{p}}\right)$ como a combinação linear:

$$
\mathrm{Y}_{1}=\mathrm{b}_{11} \mathrm{X}_{1}+\mathrm{b}_{21} \mathrm{X}_{2}+\ldots+\mathrm{b}_{\mathrm{p} 1} \mathrm{X}_{\mathrm{p}}=\mathbf{b}^{\prime}{ }_{1} \mathbf{X}
$$

cujos coeficientes $b_{i 1}$ são elementos do vetor característico $b_{1}$, associado à maior raiz característica $\left(\lambda_{1}\right)$ da matriz de covariância amostral, $\mathbf{S}$, das variáveis $\mathrm{X}_{\mathrm{i} \text { 's. }}$ O segundo componente principal $\left(\mathrm{Y}_{2}\right)$ estará associado à segunda maior raiz característica $\left(\lambda_{2}\right)$ e, assim sucessivamente, até que toda a variância tenha sido explicada. Os autovalores (ou raízes características) ordenados, ou seja, $\lambda_{1} \geq \lambda_{2} \geq \Lambda \geq \lambda_{p} \geq 0$, são as variâncias amostrais dos componentes principais. O primeiro componente também é definido como o de maior importância, uma vez que retém a maior parte da variação total encontrada nos dados originais.

A técnica envolve a matriz de covariância amostral $\mathbf{S}$ ou a matriz de correlação $\mathbf{R}$, sendo que a maioria das aplicações tem envolvido esta última (MORRISON, 1976), pois, freqüentemente, as variáveis têm escalas diferentes (idade em anos, peso em quilos, altura em metros, entre outros), devendo ser padronizadas. Após essa transformação, todas as variáveis originais têm a mesma variância unitária e, dessa maneira, a base para extração dos componentes principais é a matriz de correlação R. Segundo esse autor, a padronização dos dados é obtida por:

$$
z_{i j}=\frac{x_{i j}-\bar{x}_{j}}{s_{j}}
$$

sendo que

$\mathrm{z}_{\mathrm{ij}}$ é o valor observado padronizado da j-ésima variável no i-ésimo indivíduo, com $\mathrm{i}=1,2, \ldots, \mathrm{n}$ e $\mathrm{j}=1,2, \ldots, p ; x_{i j}$, o valor observado da j-ésima variável no i-ésimo indivíduo, com $\mathrm{i}=1,2, \ldots, \mathrm{n}$ e $\mathrm{j}=1,2, \ldots, \mathrm{p}$; $\overline{\mathrm{x}}_{\mathrm{j}}=\frac{1}{\mathrm{n}} \sum_{\mathrm{i}=1}^{\mathrm{n}} \mathrm{x}_{\mathrm{ij}}$, a estimativa da média da $\mathrm{j}$-ésima variável; $\mathrm{s}_{\mathrm{j}}=\left[\frac{1}{\mathrm{n}-1} \sum_{\mathrm{i}=1}^{\mathrm{n}}\left(\mathrm{x}_{\mathrm{ij}}-\overline{\mathrm{x}}_{\mathrm{j}}\right)^{2}\right]^{\frac{1}{2}}$, o desvio-padrão daj-ésima variável; n, número de observações; e p, número de variáveis.
Assim, tem-se: $\mathbf{R}=\mathbf{Z}^{\prime} \mathbf{Z}$, sendo $\mathbf{Z}$ a matriz dos dados padronizados.

Segundo JOLLIFFE (1972 e 1973), quando se dispõe de grande número de descritores, é possível que muitos deles sejam redundantes, tornando-se útil a sua eliminação, porque, além de pouco informativos, ocorre acréscimo no trabalho de avaliação e não apresentam informação adicional.

Com base no princípio de que a importância ou variância dos componentes principais decresce do primeiro para o último, tem-se que os últimos componentes explicam uma fração muito pequena da variância total. Então, a variável de maior coeficiente no componente de menor autovalor dever ser a menos importante para explicar a variância total e, portanto, passível de descarte (PEREIRA, 1989).

A técnica dos componentes principais tem a vantagem adicional de avaliar a importância de cada caractere estudado sobre a variação total disponível entre os acessos avaliados, possibilitando o descarte dos caracteres menos discriminantes, por já estarem correlacionados com outras variáveis ou pela sua invariância (DAHER et al., 1997).

CURY (1993) realizou o descarte das variáveis através da técnica dos componentes principais, descartando a variável com maior coeficiente do componente com menor autovalor em sucessivas análises, isto é, a cada descarte repetiu a análise considerando o novo subconjunto de variáveis.

DIAS (1994) descartou as variáveis que apresentaram os maiores elementos dos autovetores nos últimos componentes, utilizando o critério de JOLLIFFE (1972, 1973), complementado pelo procedimento usado por CURY (1993).

O critério adotado foi descartar a variável de maior coeficiente em cada componente com autovalor menor que 0,70 , de acordo com as pressuposições de JOLLIFFE (1972, 1973) e MARDIA et al. (1979).

Devido ao número pequeno de acessos disponíveis para este estudo $(n=27)$ e à grande quantidade de variáveis, optou-se por realizar uma análise de componentes principais para cada conjunto de variáveis; em seguida, procedeu-se à eliminação dos descritores redundantes.

Foi efetuada uma análise de componentes principais considerando os caracteres anteriormente selecionados, para se proceder a um novo descarte, totalizando, portanto, seis análises de componentes principais.

As estimativas das correlações entre os descritores selecionados e descartados foram obti- 
das como medida da eficiência da análise de componentes principais, para descartar os descritores redundantes.

De acordo com JOLLIFFE (1973), pode-se fazer uma comparação dos componentes principais para os conjuntos de dados completo e reduzido. O coeficiente de correlação, $r_{1}$, foi usado como medida de similaridade entre um par particular dos componentes.

Do conjunto de dados completo, algum componente principal pode ser escrito como uma combinação linear das p variáveis; assim, tem-se:

$$
\begin{gathered}
Y_{i}=a_{1 i} X_{1}+a_{2 i} X_{2}+\ldots+a_{p i} X_{p}=a^{\prime}{ }_{i} X \\
\operatorname{com~} \mathrm{i}=1,2, \ldots, p
\end{gathered}
$$

De maneira análoga, para o conjunto de dados reduzido, algum componente principal pode ser escrito como:

$$
\begin{gathered}
\mathrm{W}_{\mathrm{j}}=\mathrm{b}_{1 \mathrm{j}} \mathrm{X}_{1}+\mathrm{b}_{2 \mathrm{j}} \mathrm{X}_{2}+\ldots+\mathrm{b}_{\mathrm{pj}} \mathrm{X}_{\mathrm{p}}=\mathbf{b}_{\mathbf{j}} \mathbf{X}^{\prime} \mathbf{X} \\
\operatorname{com} \mathrm{j}=1,2, \ldots, \mathrm{p}
\end{gathered}
$$

em que os coeficientes a e b são constantes, sendo nulos os valores de $\mathrm{b}$ correspondentes às variáveis descartadas.

Assim, o coeficiente de correlação entre $\mathrm{Y}_{\mathrm{i}} \mathrm{e} \mathrm{W}_{\mathrm{j}}$, denotado $\mathrm{r}_{1}$, é dado por:

$$
r_{1}=\frac{\left(\sum_{i=1}^{p} \sum_{j=1}^{p} a_{i} b_{j} r_{i j}\right)}{\left\{\left(\sum_{i=1}^{p} \sum_{j=1}^{p} a_{i} a_{j} r_{i j}\right)\left(\sum_{i=1}^{p} \sum_{j=1}^{p} b_{i} b_{j} r_{i j}\right)\right\}^{\frac{1}{2}}}
$$

Como medida de similaridade entre dois conjuntos de componentes, ou seja, $\mathrm{Q}_{1}$, deve ser usada uma média ponderada dos $\mathrm{r}_{1}$ 's para os vários pares de componentes, obtido por:

$$
\mathrm{Q}_{1}=\frac{\sum_{\mathrm{i}=1}^{\mathrm{m}} \mathrm{q}_{\mathrm{i}} \mathrm{r}_{1}(\mathrm{j})}{\sum_{\mathrm{i}=1}^{\mathrm{m}} \mathrm{q}_{\mathrm{i}}}
$$

em que $r_{1}(j)$ é o valor máximo de $r_{1}$, definido acima, entre o i-ésimo componente para o conjunto de dados completo e algum componente para o conjunto de dados reduzido e $\mathrm{q}_{\mathrm{i}}$, a proporção da variação total explicada pelo i-ésimo componente no conjunto de dados completo.

\section{Resultados e Discussão}

A Tabela 1 apresenta as estimativas dos coeficientes de correlação entre os descritores seleciona- dos e descartados, para os descritores reprodutivos.

Os descritores selecionados que apresentaram o maior número de coeficiente de correlação significativos com os descritores descartados foram: CE (três combinações), CEF (seis combinações) e LMS (três combinações).

Estes resultados estão de acordo com a idéia apresentada anteriormente, de que os descritores descartados, por explicarem o mesmo tipo de informação que os selecionados, são redundantes, sendo, portanto, de interesse a sua eliminação.

Observa-se, na Tabela 2, que esse germoplasma foi caracterizado pelos descritores PORMS e FDN em todas as avaliações, o que não ocorreu com o descritor NOTA. Na avaliação anual e de inverno, foram descartados os mesmos descritores.

As medidas de similaridade, $r_{1}(j)$ e $Q_{1}$, entre os componentes para os conjuntos completo e reduzido dos descritores reprodutivos e agronômicos encontram-se nas Tabelas 3 e 4, respectivamente.

Observa-se, na Tabela 1, que o descritor PR não está correlacionado com nenhum dos demais, sendo o último a ser descartado, de acordo com o critério proposto. Adotando-se um valor 0,60 para os autovalores, este descritor também poderia ser selecionado.

Dessa forma, verificou-se pela Tabela 3 que a adição da variável PR provoca melhora nos valores dos coeficientes de correlação $r_{1}$ e a mesma ordenação até o terceiro componente. Para o conjunto de seis descritores selecionados, o quarto componente reduzido é uma mescla do terceiro e sexto componentes originais, e para o conjunto de sete descritores, o quinto componente reduzido é uma mescla do quarto e sexto componentes originais. Portanto, os descritores selecionados foram: PEF, DREF, EFL, CE, CEF, LMS e PR.

Observando os coeficientes de correlação $r_{1}$, da Tabela 4, verificou-se que os seus valores são altos para as três avaliações. A avaliação de verão reproduziu os componentes originais, o mesmo ocorrendo para a de inverno, apenas trocando a ordem dos componentes 1 e 2 . Na avaliação anual, o primeiro componente reduzido foi uma mescla dos componentes completos 1 e 2 , sendo que esta avaliação foi a que apresentou os menores valores para os coeficientes de correlação $r_{1}$ e $Q_{1}$. Os seguintes descritores foram considerados como selecionados: PORMSANO, PPMSANO, FDNANO, REBROAN, PRMSVER e DIVERAO.

De modo análogo, estas observações foram analisadas com as variáveis vegetativas, sendo selecio- 
Rev. bras. zootec.

Tabela 1 - Estimativas dos coeficientes de correlação entre os descritores selecionados (horizontal) e descartados (vertical) para os 15 descritores reprodutivos das espécies Paspalum guenoarum e Paspalum plicatulum - 1996

Table 1 - Estimates of correlation coefficientes among the selected (horizontal) and discarded (vertical) descriptors for the 15 reproductive descriptors of the species Paspalum guenoarum and Paspalum plicatulum - 1996

\begin{tabular}{lcccccc}
\hline Descritores & \multicolumn{5}{c}{ Descritores selecionados (Selected descriptors) } \\
\cline { 2 - 7 } descartados & $\mathrm{PEF}^{1}$ & $\mathrm{CE}^{2}$ & $\mathrm{CEF}^{3}$ & $\mathrm{DREF}^{4}$ & $\mathrm{EFL}^{5}$ & $\mathrm{LMS}^{6}$ \\
$\begin{array}{l}\text { Discarded } \\
\text { descriptors }\end{array}$ & $P F A$ & $L E$ & $L F A$ & $R F A D$ & $F T$ & $S W$ \\
\hline $\mathrm{PE}^{7}(P E)$ & & & & & & \\
PR $^{8}(P R)$ & $0,609^{* *}$ & $-0,474^{*}$ & $-0,416^{*}$ & $-0,149$ & 0,062 & $-0,036$ \\
PES $^{9}(P E)$ & 0,145 & $-0,052$ & 0,128 & $-0,318$ & 0,139 & $-0,088$ \\
NMRI $^{10}(N R I)$ & $-0,006$ & $-0,324$ & $0,392^{*}$ & 0,272 & 0,171 & $-0,020$ \\
NMPR $^{11}(N P R)$ & 0,010 & 0,091 & $0,511^{*}$ & $0,466^{*}$ & $-0,439$ & 0,001 \\
CMRML $^{12}(L L R)$ & 0,067 & 0,237 & $0,600^{* *}$ & 0,233 & $-0,446$ & 0,295 \\
EM $^{13}(M T)$ & $-0,007$ & 0,093 & $0,801^{* *}$ & 0,323 & $-0,463$ & 0,192 \\
CMS $^{14}(S L)$ & 0,050 & 0,021 & $-0,504^{* *}$ & $-0,190$ & $0,807^{* *}$ & $-0,619^{*}$ \\
NMS $^{15}(N S G)$ & $-0,068$ & $0,363^{* *}$ & 0,441 & 0,034 & $-0,527$ & $0,600^{* *}$ \\
\hline
\end{tabular}

* ** significativo em nível de 5 e $1 \%$, respectivamente, a significância foi obtida a partir do coeficiente de correlação de posto (Spearman).

*, ** significant at 5 and $1 \%$, respectively, the significance was obtained from Sperman's coefficient of rank correlation 1 Pilosidade do eixo floral (Pubescence of the floral axis).

2 Comprimento do escapo (Length of the escape).

Comprimento do eixo floral (Length of the floral axis).

4 Disposição do rácemo no eixo floral (Raceme in the floral axis disposition).

5 Época de florescimento (Flowering time).

6 Largura média da semente (Average seed width).

Pilosidade do escapo (Pubescence of the escape).

8 Pilosidade do rácemo (Pubescence of the raceme).

9 Pilosidade da espigueta (Pubescence of the spikelets).

10 Número médio de rácemo por inflorescência (Average number of racemes per inflorescence).

11 Número médio de pedicelos por rácemo (Average number of pedicel per raceme).

12 Comprimento médio do rácemo mais longo (Average length of the longest raceme).

13 Época de maturação (Maturation time).

14 Comprimento médio da semente (Average seed length).

15 Número médio de sementes em 1 grama (Average number of seeds per gram).

Tabela 2 - Descritores agronômicos das espécies Paspalum guenoarum e Paspalum plicatulum selecionados e descartados para as avaliações anuais de inverno e verão - 1996

Table 2 - Agronomic descriptors of the species Paspalum guenoarum and Paspalum plicatulum selected and discarded for the annual winter and summer evaluations - 1996

\begin{tabular}{|c|c|c|c|c|c|c|c|}
\hline \multirow[b]{2}{*}{$\begin{array}{l}\text { Avaliação } \\
\text { Evaluation }\end{array}$} & \multicolumn{7}{|c|}{$\begin{array}{l}\text { Descritores } \\
\text { Descriptors }\end{array}$} \\
\hline & $\begin{array}{c}\text { PORMS }^{1} \\
D M \\
\end{array}$ & $\begin{array}{c}\mathrm{PRMS}^{2} \\
D M Y\end{array}$ & $\begin{array}{l}\text { PPMS }^{3} \\
C P P D M\end{array}$ & $\begin{array}{c}\mathrm{FDN}^{4} \\
N D F\end{array}$ & $\begin{array}{c}\mathrm{DIV}^{5} \\
I V D M D\end{array}$ & $\begin{array}{c}\text { NOTA }^{6} \\
V D\end{array}$ & $\begin{array}{c}\text { REBROTA }^{7} \\
R G \\
\end{array}$ \\
\hline $\begin{array}{l}\text { Anual } \\
\text { Annual }\end{array}$ & $\mathrm{S}^{8}$ & $\mathrm{D}^{9}$ & S & S & D & $\mathrm{D}$ & S \\
\hline $\begin{array}{l}\text { Inverno } \\
\text { Winter }\end{array}$ & $S$ & $\mathrm{D}$ & $\mathrm{S}$ & $\mathrm{S}$ & $\mathrm{D}$ & $\mathrm{D}$ & $\mathrm{S}$ \\
\hline $\begin{array}{l}\text { Verão } \\
\text { Summer }\end{array}$ & $\mathrm{S}$ & $\mathrm{S}$ & $\mathrm{D}$ & $\mathrm{S}$ & $\mathrm{S}$ & $\mathrm{D}$ & $\mathrm{D}$ \\
\hline $\begin{array}{ll}1 & \text { Porcentage } \\
2 & \text { Produção t } \\
3 & \text { Porcentage } \\
4 & \text { Porcentage } \\
5 & \text { Porcentag } \\
6 & \text { Nota média } \\
7 & \text { Rebrota m } \\
8 & \text { Descritor s } \\
9 & \text { Descritor d }\end{array}$ & $\begin{array}{l}m \text { média de } m \\
\text { tal de matéria } \\
m \text { média de p } \\
m \text { média de fit } \\
m \text { média de di } \\
\text { de desenvolv } \\
\text { dia (regrowth [s } \\
\text { lecionado ( } \\
\text { escartado (Dis }\end{array}$ & $\begin{array}{l}\text { latéria seca } \\
\text { a seca (Dry } \\
\text { roteína brut } \\
\text { bra em det } \\
\text { igestibilidac } \\
\text { imento veg } \\
\text { score]). } \\
\text { elected desci } \\
\text { carded desc }\end{array}$ & $\begin{array}{l}\text { (Average dry } \\
\text { natter yield). } \\
\text { na matéria } \\
\text { gente neutr } \\
\text { e in vitro (Av } \\
\text { tativo (Vege } \\
\text { ptors). } \\
\text { iptors). }\end{array}$ & $\begin{array}{l}\text { natter perce } \\
\text { seca (Aver } \\
\text { (Average } \\
\text { rage in vitrc } \\
\text { ative develo }\end{array}$ & $\begin{array}{l}\text { age). } \\
\text { ue crude prot } \\
\text { try matterg dic } \\
\text { ment [score] }\end{array}$ & $\begin{array}{l}\text { in percentage } \\
\text { t fiber percen } \\
\text { stibility perce }\end{array}$ & $\begin{array}{l}\text { in dry matter). } \\
\text { tage). } \\
\text { ntage). }\end{array}$ \\
\hline
\end{tabular}


STRAPASSON et al.

Tabela 3 - Medidas de similaridade, $r_{1}(j)$ e $Q_{1}$, entre componentes para os conjuntos completo e reduzido dos descritores reprodutivos das espécies Paspalum guenoarum e Paspalum plicatulum com seis e sete descritores selecionados - 1996

Table 3 - Measures of similarity, $r_{1}(j)$ and $Q_{1}$, between components for the overall and for reduced set of the reproductive descriptors of the species Paspalum guenoarum and Paspalum plicatulum with six and seven selected descritors - 1996

\begin{tabular}{lcc}
\hline & \multicolumn{2}{c}{ Critério de rejeição (Rejection criterion) } \\
\cline { 2 - 3 } Medidas de similidade & 0,70 & 0,60 \\
\cline { 2 - 3 } & \multicolumn{2}{c}{ Descritores selecionados (Selected descriptors) } \\
\cline { 2 - 3 } & $\mathrm{PEF}^{1}(P F A), \mathrm{DREF}^{2}($ RFAD) & $\mathrm{PEF}^{1}(P F A), \mathrm{DREF}^{2}(R F A D)$, \\
& $\mathrm{PEFL}^{3}(F T), \mathrm{CE}^{4}(L E)$ & $\mathrm{EFL}^{3}(F T), \mathrm{CE}^{4}(L E)$ \\
$\mathrm{CEF}^{5}(L F A), \mathrm{LMS}^{6}(S W)$ & $\mathrm{CEF}^{5}(L F A), \mathrm{LMS}^{6}(S W), \mathrm{PR}^{7}(P R)$ \\
$\mathrm{r}_{1}(1)$ & $0,631(1)^{+}$ & $0,663(1)$ \\
$\mathrm{r}_{1}(2)$ & $0,721(2)$ & $0,818(2)$ \\
$\mathrm{r}_{1}(3)$ & $0,549(4)$ & $0,710(3)$ \\
$\mathrm{r}_{1}(4)$ & $0,482(5)$ & $0,518(5)$ \\
$\mathrm{r}_{1}(5)$ & $0,681(3)$ & $0,775(4)$ \\
$\mathrm{r}_{1}(6)$ & $0,628(4)$ & $0,605(5)$ \\
$\mathrm{r}_{1}(7)$ & - & $0,772(7)$ \\
\hline $\mathrm{Q}_{1}$ & 0,626 & 0,694 \\
\hline
\end{tabular}

+ Entre parênteses: número do componente do conjunto de dados reduzido mais altamente correlacionado com o j-ésimo componente do conjunto de dados completo.

In brackets: number of the component from the reduced set of data most highly correlated with the jth component from the full set of data.

1 Pilosidade do eixo floral (Pubescence of the floral axis).

2 Disposição do rácemo no eixo floral (Raceme in the floral axis disposition).

3 Época de florescimento (Flowering time).

4 Comprimento do escapo (Length of the escape).

5 Comprimento do eixo floral (Length of the floral axis).

6 Largura média da semente (Seed width [mean]).

7 Pilosidade do rácemo (Pubescence of the raceme).

Tabela 4 - Medidas de similaridade $r_{1}(j)$ e $Q_{1}$ entre componentes para os conjuntos completo e reduzido dos descritores agronômicos das espécies Paspalum guenoarum e Paspalum plicatulum - 1996

Table 4 - Measures of similarity, $r_{1}(j)$ and $Q_{1}$, between components for the overall and for reduced set of the agronomic descriptors of the species Paspalum guenoarum and Paspalum plicatulum - 1996

\begin{tabular}{|c|c|c|c|}
\hline \multirow{5}{*}{$\begin{array}{l}\text { Medidas de } \\
\text { Measures of }\end{array}$} & \multicolumn{3}{|c|}{$\begin{array}{l}\text { Avaliação } \\
\text { Evaluation }\end{array}$} \\
\hline & Anual & Inverno & Verão \\
\hline & Annual & Winter & Summer \\
\hline & \multirow{2}{*}{\multicolumn{3}{|c|}{$\begin{array}{l}\text { Descritores selecionados } \\
\text { Selected descriptors }\end{array}$}} \\
\hline & & & \\
\hline \multirow{4}{*}{$\begin{array}{l}\text { Similaridade } \\
\text { Similarity }\end{array}$} & $\operatorname{PORMS}^{1}(D M)$ & $\operatorname{PORMS}^{1}(D M)$ & $\operatorname{PORMS}^{1}(D M)$ \\
\hline & $\mathrm{PPMS}^{2}(C P P D M)$, & $\operatorname{PPMS}^{2}(C P P D M)$, & $\mathrm{FDN}^{3}(N D F)$, \\
\hline & $\mathrm{FDN}^{3}(N D F)$ & $\mathrm{FDN}^{3}(N D F)$ & $\operatorname{PRMS}^{5}(D M Y)$, \\
\hline & REBROTA $^{4}(R G)$ & $\operatorname{REBROTA}^{4}(R G)$ & $\mathrm{DIV}^{6}(I V D M D)$ \\
\hline$\overline{r_{1}(1)}$ & $0,689(1)^{+}$ & $0,722(2)$ & $0,858(1)$ \\
\hline$r_{1}(2)$ & $0,696(1)$ & $0,767(1)$ & $0,745(2)$ \\
\hline$r_{1}(3)$ & $0,893(3)$ & $0,985(3)$ & $0,814(3)$ \\
\hline$r_{1}(4)$ & $0,869(4)$ & $0,923(4)$ & $0,897(4)$ \\
\hline $\mathrm{Q}_{1}$ & 0,730 & 0,779 & 0,826 \\
\hline \multicolumn{4}{|c|}{$\begin{array}{l}\text { Entre parênteses: número do componente do conjunto de dados reduzido mais altamente correlacionado } \\
\text { com o j-ésimo componente do conjunto de dados completo } \\
\text { In brackets:number of the component from reduced setof data mosthighly correlated with thejth componentfrom the overal } \\
\text { set of data. } \\
1 \text { Porcentagem média de matéria seca (Dry matter percentage [mean]). } \\
2 \text { Porcentagem média de proteína bruta na matéria seca (Crude protein percentage in dry matter [mean]). } \\
3 \text { Porcentagem média de fibra em detergente neutro (Neutral detergent fiber percentage [mean]). } \\
4 \text { Rebrota média (Regrowth [escore]). } \\
5 \text { Produção total de matéria seca (Dry matter yield). } \\
6 \text { Porcentagem média de digestibilidade in vitro (in vitro dry matter digestibility [mean]). }\end{array}$} \\
\hline
\end{tabular}


Rev. bras. zootec.

nados os seguintes descritores: COPLF, DIPB, CMLF, LMLFMEIO, CMB, LMBBASE e CMEN.

Após a seleção de descritores reprodutivos, vegetativos e agronômicos, separadamente, foi reali- zada uma análise de componentes principais considerando estes 20 descritores conjuntamente (Tabela 5), a fim de se proceder à nova seleção; posteriormente, foram obtidas as medidas de similaridade $\mathrm{r}_{1}$ e $\mathrm{Q}_{1}$.

Tabela 5 - Estimativas das variâncias (autovalor $\lambda_{\mathrm{j}}$ ), variância acumulada (\%) e autovetores dos 20 descritores botânico-agronômicos selecionados em acessos das espécies Paspalum guenoarum e Paspalum plicatulum - 1996

Table 5 - Estimates of the variances (eigenvalues $\lambda_{j}$ ), accumulated variance (\%) and eigenvectors of the 20 botanicalagronomic descriptors selected in accessions of the species Paspalum guenoarum and Paspalum plicatulum - 1996

\begin{tabular}{|c|c|c|c|c|c|c|c|c|}
\hline \multirow[t]{2}{*}{$\lambda_{\mathrm{j}}$} & \multirow{2}{*}{$\begin{array}{l}\text { Variância } \\
\text { acumulada } \\
\text { Acumulated } \\
\text { variance }\end{array}$} & \multicolumn{7}{|c|}{$\begin{array}{c}\text { Descritores } \\
\text { Descriptors }\end{array}$} \\
\hline & & $\begin{array}{c}\mathrm{PEF}^{1} \\
P F A\end{array}$ & $\begin{array}{l}\mathrm{PR}^{2} \\
P R\end{array}$ & $\begin{array}{l}\mathrm{CE}^{3} \\
L E\end{array}$ & $\begin{array}{c}\mathrm{CEF}^{4} \\
L F A\end{array}$ & $\begin{array}{c}\mathrm{DREF}^{5} \\
R F A D\end{array}$ & $\begin{array}{c}\mathrm{EFL}^{6} \\
F T\end{array}$ & $\begin{array}{c}\mathrm{LMS}^{7} \\
S W\end{array}$ \\
\hline 5,348 & 26,738 & 0,012 & $-0,003$ & $-0,009$ & 0,372 & 0,254 & $-0,206$ & 0,084 \\
\hline 3,082 & 42,146 & 0,319 & 0,051 & 0,306 & $-0,133$ & 0,053 & 0,158 & 0,225 \\
\hline 2,857 & 56,429 & $-0,077$ & 0,100 & 0,303 & $-0,147$ & $-0,112$ & 0,084 & $-0,208$ \\
\hline 2,246 & 67,657 & 0,311 & $-0,150$ & $-0,342$ & 0,063 & 0,262 & 0,169 & $-0,429$ \\
\hline 1,760 & 76,456 & $-0,178$ & $-0,588$ & 0,116 & $-0,178$ & 0,388 & $-0,322$ & 0,250 \\
\hline 1,031 & 81,610 & 0,268 & 0,092 & $-0,250$ & 0,024 & $-0,069$ & $-0,238$ & 0,119 \\
\hline 0,877 & 85,996 & $-0,493$ & 0,145 & $-0,131$ & $-0,030$ & 0,186 & 0,528 & 0,107 \\
\hline 0,721 & 89,601 & 0,210 & 0,507 & $-0,077$ & 0,052 & 0,050 & $-0,228$ & $-0,120$ \\
\hline 0,586 & 92,533 & 0,124 & 0,216 & 0,068 & $-0,107$ & 0,147 & 0,240 & 0,060 \\
\hline 0,455 & 94,809 & 0,206 & 0,048 & $-0,124$ & $-0,175$ & $-0,344$ & $-0,170$ & 0,468 \\
\hline 0,341 & 96,513 & $-0,142$ & 0,338 & 0,326 & $-0,288$ & 0,179 & $-0,078$ & 0,087 \\
\hline 0,206 & 97,545 & 0,033 & $-0,307$ & 0,106 & 0,228 & $-0,486$ & 0,282 & 0,000 \\
\hline 0,163 & 98,360 & 0,398 & $-0,164$ & 0,296 & $-0,160$ & $-0,056$ & 0,315 & $-0,015$ \\
\hline 0,126 & 98,987 & 0,336 & $-0,015$ & 0,172 & 0,063 & 0,458 & 0,152 & 0,173 \\
\hline 0,104 & 99,509 & 0,118 & 0,019 & $-0,249$ & 0,082 & 0,033 & 0,167 & 0,381 \\
\hline 0,056 & 99,791 & $-0,060$ & $-0,032$ & 0,253 & 0,078 & $-0,169$ & $-0,127$ & 0,023 \\
\hline 0,017 & 99,878 & $-0,098$ & 0,169 & 0,356 & 0,639 & 0,014 & $-0,051$ & 0,164 \\
\hline 0,014 & 99,950 & 0,124 & $-0,102$ & 0,034 & 0,329 & 0,058 & 0,148 & 0,076 \\
\hline 0,009 & 99,997 & $-0,025$ & 0,047 & $-0,153$ & 0,199 & 0,065 & 0,100 & 0,080 \\
\hline 0,001 & 100,000 & $-0,107$ & 0,077 & $-0,258$ & $-0,080$ & $-0,028$ & 0,183 & 0,409 \\
\hline
\end{tabular}

1 Pilosidade do eixo floral (Pubescence of the floral axis).

2 Pilosidade do rácemo (Pubescence of the raceme).

3 Comprimento do escapo (Length of the escape).

4 Comprimento do eixo floral (Length of the floral axis).

5 Disposição do rácemo no eixo floral (Raceme in the floral axis disposition).

6 Época de florescimento (Flowering time).

7 Largura média da semente (Seed width [mean]).

A Tabela 5 continua na próxima página... 
STRAPASSON et al.

Continuação da Tabela 5

\begin{tabular}{|c|c|c|c|c|c|c|c|c|}
\hline \multirow[t]{2}{*}{$\lambda_{j}$} & \multirow{2}{*}{$\begin{array}{c}\text { Variância } \\
\text { Acumulada } \\
\text { Acumulated } \\
\text { variance }\end{array}$} & \multicolumn{7}{|c|}{ Descritores (Descriptors) } \\
\hline & & $\begin{array}{c}\text { COPLF }^{8} \\
P L L\end{array}$ & $\begin{array}{c}\mathrm{DIPB}^{9} \\
P S D\end{array}$ & $\begin{array}{c}\mathrm{CMLF}^{10} \\
L L B\end{array}$ & $\begin{array}{c}\mathrm{LMLF}^{11} \\
M E I O \\
L W H \\
\end{array}$ & $\begin{array}{c}\mathrm{CMB}^{12} \\
S L\end{array}$ & $\begin{array}{c}\mathrm{LMB}^{13} \\
B A S E \\
S W\end{array}$ & $\begin{array}{c}\mathrm{CMEN}^{14} \\
I L\end{array}$ \\
\hline 5,348 & 26,738 & $-0,126$ & $-0,009$ & 0,337 & 0,302 & 0,361 & 0,301 & 0,179 \\
\hline 3,082 & 42,146 & 0,375 & $-0,218$ & $-0,254$ & 0,128 & $-0,210$ & $-0,079$ & 0,005 \\
\hline 2,857 & 56,429 & 0,142 & $-0,035$ & 0,151 & $-0,322$ & 0,191 & $-0,349$ & 0,328 \\
\hline 2,246 & 67,657 & 0,152 & 0,337 & $-0,038$ & $-0,155$ & $-0,012$ & $-0,153$ & $-0,273$ \\
\hline 1,760 & 76,456 & 0,237 & 0,246 & $-0,023$ & $-0,083$ & $-0,051$ & $-0,044$ & 0,170 \\
\hline 1,031 & 81,610 & 0,347 & $-0,530$ & 0,232 & $-0,119$ & 0,076 & $-0,014$ & $-0,126$ \\
\hline 0,877 & 85,996 & $-0,073$ & $-0,097$ & $-0,008$ & 0,127 & 0,019 & $-0,008$ & $-0,184$ \\
\hline 0,721 & 89,601 & 0,150 & 0,439 & 0,010 & 0,066 & $-0,029$ & 0,047 & 0,114 \\
\hline 0,586 & 92,533 & 0,122 & 0,033 & $-0,011$ & 0,198 & $-0,077$ & 0,203 & 0,543 \\
\hline 0,455 & 94,809 & $-0,358$ & 0,179 & $-0,067$ & $-0,104$ & 0,075 & $-0,089$ & $-0,089$ \\
\hline 0,341 & 96,513 & 0,123 & 0,201 & 0,309 & $-0,274$ & $-0,111$ & 0,344 & $-0,385$ \\
\hline 0,206 & 97,545 & 0,425 & 0,197 & 0,331 & 0,154 & 0,029 & 0,226 & $-0,118$ \\
\hline 0,163 & 98,360 & $-0,314$ & 0,167 & 0,020 & $-0,039$ & 0,321 & 0,082 & 0,053 \\
\hline 0,126 & 98,987 & $-0,078$ & $-0,161$ & 0,079 & 0,072 & 0,206 & $-0,113$ & $-0,312$ \\
\hline 0,104 & 99,509 & 0,050 & 0,233 & $-0,069$ & 0,168 & $-0,370$ & $-0,016$ & 0,034 \\
\hline 0,056 & 99,791 & 0,040 & 0,057 & $-0,362$ & 0,051 & 0,077 & 0,289 & $-0,282$ \\
\hline 0,017 & 99,878 & 0,046 & 0,152 & $-0,102$ & $-0,057$ & $-0,004$ & $-0,447$ & $-0,071$ \\
\hline 0,014 & 99,950 & $-0,258$ & $-0,108$ & 0,231 & $-0,550$ & $-0,464$ & 0,222 & 0,161 \\
\hline 0,009 & 99,997 & 0,201 & $-0,037$ & $-0,526$ & $-0,427$ & 0,354 & 0,379 & 0,119 \\
\hline 0,001 & 100,000 & 0,197 & 0,185 & 0,225 & $-0,222$ & 0,354 & $-0,198$ & 0,053 \\
\hline
\end{tabular}

8 Comprimento dos pêlos no limbo foliar(Pubescence of the leaf-length).

9 Distribuição dos pêlos nas bainhas (Pubescence in the sheath-distribution).

10 Comprimento médio da lâmina foliar da base (Leafblades length-base [mean]).

11 Largura média da lâmina foliar do meio (Leafblades width-half [mean]).

12 Comprimento médio da bainha (Sheath length [mean]).

13 Largura média da base da bainha (Sheath width [mean]).

14 Comprimento médio dos entrenós (Internode length [mean]).

Continuação da Tabela 5

\begin{tabular}{|c|c|c|c|c|c|c|c|}
\hline \multirow[t]{4}{*}{$\bar{\lambda}_{\mathrm{j}}$} & \multirow{4}{*}{$\begin{array}{l}\text { Variância } \\
\text { acumulada } \\
\text { Acumulated } \\
\text { variance }\end{array}$} & \multicolumn{6}{|c|}{ Descritores (Descriptors) } \\
\hline & & PORMS & PPMS & FDN & REBRO & PRMS & DIVERAO \\
\hline & & $A N O^{15}$ & $A N O^{16}$ & $A N O^{17}$ & $A N O^{18}$ & $V E R^{19}$ & \\
\hline & & $D M$ & $C P P D M$ & $N D F$ & $R G$ & $D M Y$ & $I V D M D$ \\
\hline 5,348 & 26,738 & $-0,127$ & 0,166 & $-0,149$ & 0,291 & 0,271 & 0,223 \\
\hline 3,082 & 42,146 & $-0,276$ & 0,378 & $-0,290$ & 0,059 & 0,077 & 0,271 \\
\hline 2,857 & 56,429 & 0,347 & $-0,147$ & $-0,192$ & 0,324 & 0,316 & 0,104 \\
\hline 2,246 & 67,657 & $-0,038$ & 0,254 & 0,202 & 0,184 & 0,277 & 0,040 \\
\hline 1,760 & 76,456 & 0,195 & 0,033 & 0,120 & $-0,060$ & $-0,131$ & 0,170 \\
\hline 1,031 & 81,610 & 0,143 & $-0,166$ & 0,410 & $-0,001$ & $-0,045$ & 0,269 \\
\hline 0,877 & 85,996 & 0,309 & 0,176 & 0,109 & 0,015 & $-0,081$ & 0,428 \\
\hline 0,721 & 89,601 & 0,295 & 0,008 & $-0,218$ & $-0,318$ & $-0,244$ & 0,301 \\
\hline 0,586 & 92,533 & 0,137 & 0,152 & 0,510 & $-0,051$ & 0,132 & $-0,345$ \\
\hline 0,455 & 94,809 & 0,285 & 0,422 & 0,088 & 0,279 & 0,020 & $-0,030$ \\
\hline 0,341 & 96,513 & $-0,187$ & $-0,036$ & 0,118 & 0,256 & $-0,029$ & $-0,097$ \\
\hline 0,206 & 97,545 & 0,206 & 0,095 & $-0,076$ & 0,042 & $-0,184$ & $-0,120$ \\
\hline 0,163 & 98,360 & $-0,184$ & $-0,270$ & 0,242 & $-0,075$ & $-0,195$ & 0,387 \\
\hline 0,126 & 98,987 & 0,398 & $-0,101$ & $-0,236$ & $-0,124$ & $-0,057$ & $-0,389$ \\
\hline 0,104 & 99,509 & 0,015 & $-0,599$ & $-0,094$ & 0,333 & 0,181 & 0,066 \\
\hline 0,056 & 99,791 & 0,221 & $-0,114$ & 0,128 & $-0,310$ & 0,621 & 0,109 \\
\hline 0,017 & 99,878 & $-0,121$ & 0,021 & 0,344 & 0,057 & $-0,127$ & $-0,017$ \\
\hline 0,014 & 99,950 & 0,088 & 0,126 & $-0,095$ & $-0,228$ & 0,106 & 0,115 \\
\hline 0,009 & 99,997 & 0,014 & $-0,044$ & $-0,106$ & 0,229 & $-0,237$ & $-0,083$ \\
\hline 0,001 & 100,000 & $-0,321$ & 0,011 & $-0,092$ & $-0,430$ & 0,251 & $-0,087$ \\
\hline
\end{tabular}

\footnotetext{
15 Porcentagem média de matéria seca - anual (Dry matter percentage [mean] - annual).

16 Porcentagem média de proteína bruta na matéria seca - anual (Crude protein percentage in dry matter [mean] - annual).

17 Porcentagem média de fibra em detergente neutro - anual (Neutral detergent fiber [mean] - annual).

18 Rebrota média - anual (Regrowth [score] - annual).

19 Produção total de matéria seca - verão (Dry matter yield - summer).

20 Porcentagem média de digestibilidade in vitro - verão (In vitro dry matter digestibility [mean] - summer).
} 


\section{Conclusões}

Houve descarte de $86 \%$ do total de descritores utilizados na caracterização dos acessos, evidenciando a necessidade de não se trabalhar com número excessivo de descritores com este tipo de material. Foram considerados como prioritários, para compor a relação de descritores, os seguintes caracteres: PR (pilosidade do rácemo), $\mathrm{CE}$ (comprimento do escapo), EFL (época de florescimento) (reprodutivos), DIPB (distribuição dos pêlos nas bainhas), CMB (comprimento médio da bainha), LMBBASE (largura média da base da bainha) (vegetativos), PORMSANO (porcentagem média de matéria seca - anual), e DIVERAO (porcentagem média de digestibilidade in vitro - verão) (agronômicos).

Os descritores agronômicos, por serem mais influenciados por fatores ambientais, tiveram menor participação na caracterização dos acessos, uma vez que, dos oito descritores selecionados, três foram reprodutivos e três vegetativos.

\section{Agradecimento}

Ao Centro de Pesquisa de Pecuária do Sudeste CPPSE/EMBRAPA, pela cessão dos dados usados neste trabalho.

Ao Conselho Nacional de Desenvolvimento Científico e Tecnológico-CNPq, pela concessão da bolsa de estudos.

\section{Referências Bibliográficas}

CHASE, A. 1929. The north American species of Paspalum. Contributions from the United States National Herbarium, 28(1):1-30.

CURY, R. Dinâmica evolutiva e caracterização de germoplasma de mandioca (Manihot esculenta Crantz) na agricultura autóctone do sul do Estado de São Paulo. Piracicaba, SP: ESALQ, 1993. 103p. Dissertação (Mestrado) - Escola Superior de Agricultura "Luiz de Queiroz”/Universidade de São Paulo, 1993.
DAHER, R.F., MORAES, C.F., CRUZ, C.D. et al. 1997. Seleção de caracteres morfológicos em capim-elefante (Pennisetum purpureum Schum.). R. Bras. Zootec., 26(2):247-259.

DIAS, L.A.S. Divergência genética e fenética multivariada na predição de híbrido e preservação de germoplasma de cacau (Theobroma cacao L.). Piracicaba, SP:ESALQ, 1994. 94p. Tese (Doutorado) - Escola Superior de Agricultura "Luiz de Queiroz"/Universidade de São Paulo, 1994.

JOHNSON, R.A., WICHERN, D.W. 1982. Apllied multivariate statistical analysis. Englewood Cliffs: Prentice Hall. 593p.

JOLLIFFE, I.T. 1972. Discarding variables in a principal component analysis. I. Artificial data. Appl. Stat., 21(2):160-173.

JOLLIFFE, I.T. 1973. Discarding variables in a principal component analysis. II. Real data. App. Stat., 21(1):21-31.

MARDIA, K.V., KENT, J.T., BIBBY, J.M. 1979. Multivariate analysis. London: Academic Press. 520p.

MORRISON, D.F. 1976. Multivariate statistical methods. 2.ed. Singapore: McGraw Hill. 415p.

PEREIRA, A.V. Utilização de análise multivariada na caracterização de germoplasma de mandioca (Manihot esculenta Crantz). Piracicaba, SP: ESALQ, 1989. 180p. Tese (Doutorado em Melhoramento e Genética) - Escola Superior de Agricultura “Luiz de Queiroz”/Universidade de São Paulo, 1989.

Recebido em: 11/03/99

Aceito em: 26/08/99 